\title{
No evidence of prenatal diversifying selection at locus or supertype levels in the dog MHC class II loci
}

\author{
Alina K. Niskanen ${ }^{1,2^{*}}$, Lorna J. Kennedy ${ }^{3}$, Hannes Lohi ${ }^{4}$, Jouni Aspi ${ }^{1}$ and Tanja Pyhäjärvi ${ }^{1}$
}

\begin{abstract}
Background: Despite decades of studying, the mechanisms maintaining high diversity in the genes of the Major Histocompatibility Complex (MHC) are still puzzling scientists. In addition to pathogen recognition and other functions, MHC molecules may act prenatally in mate choice and in maternal-foetal interactions. These interactions are potential selective mechanisms that increase genetic diversity in the MHC. During pregnancy, immune response has a dual role: the foetus represents foreign tissue compared to mother, but histo-incompatibility is required for successful pregnancy. We have studied the prenatal selection in MHC class II loci (DLA-DQA1, DLA-DQB1 and DLA-DRB1) in domestic dogs by comparing the observed and expected offspring genotype proportions in 110 dog families. Several potential selection targets were addressed, including the peptide-binding site, the MHC locus, three-locus haplotype and supertype levels. For the supertype analysis, the first canine supertype classification was created based on in silico analysis of peptide-binding amino-acid polymorphism.

Results: In most loci and levels, no deviation from the expected genotype frequencies was observed. However, one peptide-binding site in DLA-DRB1 had an excess of heterozygotes among the offspring. In addition, if the father shared a DLA-DRB1 allele with the mother, that allele was inherited by the offspring more frequently than expected, suggesting the selective advantage of a histo-compatible foetus, in contrast to our expectations.

Conclusions: We conclude that there is some evidence of post-copulatory selection at nucleotide site level in the MHC loci of pet dogs. But due to no indication of selection at locus, three-locus, or supertype levels, we estimated that the prenatal selection coefficient is less than 0.3 in domestic dogs and very likely other factors are more important in maintaining the genetic diversity in $\mathrm{MHC}$ loci.
\end{abstract}

Keywords: Canis familiaris, MHC, Prenatal selection, Supertype classification

\section{Plain English summary}

Major Histocompatibility Complex (MHC) is a large genetic region coding for immune defense molecules and it is found in all vertebrates. MHC molecules trigger immune response by binding molecules derived from pathogens and recognise self-tissues from non-self-tissues. The MHC molecules are often very diverse. This diversity is mainly thought to be a consequence of balancing

\footnotetext{
* Correspondence: alina.niskanen@gmail.com

'Department of Genetics and Physiology, University of Oulu, PO Box 3000, Oulu FIN-90014, Finland

2Present address: Centre for Biodiversity Dynamics, Department of Biology, Norwegian University of Science and Technology, NO -7491, Trondheim, Norway

Full list of author information is available at the end of the article
}

selection caused by pathogen pressure. However, also selection before birth that may take place during mate choice or during pregnancy have been demonstrated in some species. Since domestic dogs are often under intense veterinary care, pathogen pressure might be less strong compared to wild animals. Yet, selective patterns of MHC diversity before birth have not been studied before in dogs.

We studied the selection before birth at three MHC class II genes in $10 \mathrm{dog}$ breeds and four mixed-breed groups. We compared e.g. the expected and observed ratios of heterozygous and homozygous puppies in 110 dog families. We conducted selection analyses in many different levels, including: I) sites that bind molecules derived from pathogens (= peptide-binding site), II) the 
MHC genes, III) three-gene combinations and IV) supertypes. We created the first canine supertype classification that joins different MHC alleles into larger supertypes based on their chemical similarities.

We found that puppies were more heterozygous than expected at one peptide-binding site of one MHC gene (DLA-DRB1). We also found that if the father shared a DLA-DRB1 allele with the mother, that allele was inherited by the offspring more frequently than expected. Other levels of investigation did not show deviations from expected ratios of homozygous and heterozygous offspring. As a conclusion, we found some evidence of selection before birth in dogs. However, other factors are likely contributing more to the MHC diversity in dogs.

\section{Background}

Natural selection in the genes of the Major Histocompatibility Complex (MHC) has been studied extensively in wild animal populations [1]. Across many species, the MHC genes are the most polymorphic in the genome. The main function of classical MHC molecules is to bind antigenic peptides and present them to T-cells [2]. The MHC genes are divided into two classes; class I molecules are expressed on the surface of almost all nucleated cells, while, in man, class II molecules are expressed on specific antigen-presenting cells (e.g. macrophages and lymphocytes). In man, the extended MHC region also includes non-classical genes that have diverse immune and non-immune functions, including the complement system, and olfactory receptor (OR) genes $[3,4]$.

Since the function of MHC molecules is triggering immune response, parasites and diseases have been thought to play the major role in the maintenance of MHC diversity [5, 6]. However, in domesticated animals pathogen pressure has probably had a smaller contribution to the recent selection targeting $\mathrm{MHC}$, compared to wild species. Instead, there are other potential forces driving selection, such as mate choice, sperm selection, maternal-foetal interactions and autoimmune diseases [7-9]. Many MHC associations with autoimmune diseases have been identified in man [10]. Similar MHC associations have also been demonstrated in canine autoimmune disorders [11]. These associations may be one of the selective forces influencing MHC diversity among dogs (Canis familiaris).

The close relationship between the owner and their dog has been a key driver in acquiring extensive knowledge of canine diseases and resulted in a high level of veterinary care, especially in Western societies. The formation of dog breeds and artificial selection by humans has been especially strong during the last 100-150 years. As a consequence, dog breeds have become small and genetically distinct groups. MHC diversity differs from breed to breed and is often low [12], probably due to drift effects in small populations. However, even without free mate choice, MHC diversity in a dog breed can be close to the diversity of a wild wolf population [13, 14]. Humans have had major impact in some of the aspects that may influence the dog MHC diversity, for instance, through veterinary care and mate choice. Therefore, if MHC incompatibility causes prenatal selection, it may be possible to detect such selection in the domestic dog, where other factors of natural selection may be less strong.

\section{Prenatal selection}

Although the overall MHC diversity of dogs has been studied widely $[12,15]$, the role of prenatal selection in the maintenance of the MHC diversity has not previously been examined. Mate choice in dogs is more often decided by the owner rather than the dog, especially in the purebred dog population. Hence, natural selection may have its strongest impact on the MHC diversity at the post-copulatory stages from egg fertilisation until birth. Association between MHC variation and fertilisation success has been demonstrated in other species, e.g. mice [16]. Prenatal selection can be detected from non-Mendelian proportions of MHC genotypes in the offspring. For instance, the excess of heterozygous offspring [17] and increased foetal loss among MHCmatching couples [8] has been found in humans and in pigtailed macaques [18].

From the mother's perspective, a foetus corresponds to semi allograft with half of its genome being foreign. To maintain gestation, the immune response of the mother is down-regulated. However, a successful pregnancy requires some degree of maternal immune stimulation, which could be induced by histo-incompatibility (immunological dissimilarity) between the mother and the foetus $[19,20]$.

MHC molecules have crucial role in successful pregnancy. In humans, most classical MHC molecules are downregulated and only one classical MHC class I gene (HLA-C) and two nonclassical MHC class I genes (HLA-E and -G) are expressed in the foetal side of the placenta, in the extravillous trophoblast (EVT) cells [21-23]. The EVT cells migrate into the mother's uterus and transform small arteries into large blood vessels to ensure sufficient nourishment of the foetus [24]. The process of transforming the arteries is controlled by the maternal natural killer (NK) cells. Most likely all three MHC molecules, HLA-C, $-\mathrm{E}$ and $-\mathrm{G}$, interact with maternal NK cells in regulating placental vascularisation [25]. HLA-C molecule is the only polymorphic MHC molecule expressed in the EVT cells, so the success of pregnancy is influenced by the compatibility of the foetal HLA-C molecules and maternal NK cell receptors. Incongruity of the foetal MHC molecules and maternal NK cell 
receptors might lead to insufficient blood supply to the placenta which can cause pre-eclampsia, miscarriage and other pregnancy disorders [26].

The expression of MHC genes during pregnancy and mechanisms of maternal-foetal interactions in dogs are largely unknown. It has been shown that some classical class I (DLA-88) and II (DLA-DRA) MHC molecules are not expressed in early canine embryos [27] but there is an increase of MHC class II expressing cells in the uterus of pregnant females [28].

\section{MHC supertypes}

The peptide recognition repertoires of MHC molecules produced by different alleles at the same MHC locus overlap with each other to some extent. As part of the effort to develop epitope-based vaccines with the ability to immunise human populations with diverse MHC genotypes [29], MHC alleles have been grouped into supertypes, based on their structural features or peptidebinding specificity. If the actual differences in pathogen recognition and peptide binding are at the MHC supertype level, they might represent the unit of natural selection and should be taken into account when studying selection in the MHC genes. Until now, supertype classifications have mainly been produced for primates and birds [30-32], but none have been attempted for canines.

\section{Aims of the study}

The aim of this study was to investigate possible prenatal selection in three canine MHC class II loci, DLA-DRB1, DLA-DQA1, and DLA-DQB1, by examining the MHC genotype proportions in dog families. We also analysed the MHC allele composition and heterozygosity of the offspring relative to their mothers, to study potential maternal-foetal interactions. Since successful pregnancy requires histo-incompatibility between the mother and the foetus, we would expect to find an excess of heterozygous offspring under the influence of prenatal selection. Similarly, we would expect that, when parents share the same allele, the offspring will inherit the paternal non-shared allele more often than shared allele from their father. Finally, we compared the functional similarities of the identified canine alleles, and generated the first supertype classification for DLA-DRB1 and DLA-DQB1 alleles.

\section{Methods}

\section{Samples and DNA techniques}

The data comprise 430 puppies and 220 parents in 110 families that belong to ten purebred and four different mixed-breed groups (Table 1 and Additional file 1: Table S1). Each family includes parents and 1-16 puppies with the mean of 3.9 puppies. Apart from a few litters that were fully sampled, a random sample of the offspring
Table 1 The dog breeds, the number of offspring $\left(\mathrm{N}_{\mathrm{O}}\right)$, the number of families $\left(\mathrm{N}_{\mathrm{F}}\right)$, the country of sampling and the $\mathrm{MHC}$ genotyping method

\begin{tabular}{lllll}
\hline Breed & $N_{O}$ & $N_{F}$ & Country $^{\text {a }}$ & $\begin{array}{l}\text { MHC genotyping } \\
\text { method }\end{array}$ \\
\hline Kooikerhondje $^{b}$ & 17 & 10 & Finland & Sequencing \\
Saluki $^{b}$ & 51 & 16 & Finland & Sequencing \\
Lowchen $^{b}$ & 9 & 4 & Finland & Sequencing \\
Icelandic Sheepdog $^{b}$ & 7 & 3 & Finland & Sequencing \\
Kromfohrlander $^{\text {b }}$ & 90 & 24 & Finland & Sequencing \\
Alaskan Husky $^{c}$ & 27 & 6 & USA & Sequencing \\
Alaskan Husky $^{d}$ & 4 & 1 & USA & Sequencing \\
English Cocker Spaniel $^{d}$ & 6 & 1 & USA & Sequencing \\
Golden Retriever $^{d}$ & 7 & 3 & USA & Sequencing \\
Staffordshire Bull Terrier $^{d}$ & 7 & 3 & USA & Sequencing \\
Newfoundland $^{d}$ & 42 & 12 & UK & Sequencing \\
Rhodesian Ridgeback $^{d}$ & 42 & 5 & UK & Sequencing \\
Mixed-breed $^{d}$ & 84 & 16 & USA & Sequencing \\
Mixed-breed $^{c}$ & 37 & 6 & USA & Sequencing or \\
& & & & microsatellite based \\
Total $^{430}$ & 110 & &
\end{tabular}

${ }^{\mathrm{a} B i r t h}$ country may differ from the country of sampling, ${ }^{\mathrm{b}}$ Data from this study, 'Rowan [35], dKennedy et al. [34]

was available for most families. Multiple litters from the same parents were combined as one litter. The dogs were sampled in Finland, UK and USA. Thirty-six individuals were in the dataset both as a parent and as an offspring. Thirty parents had more than one litter with different mates (24 parents had two, four had three, and two had four litters).

The highly polymorphic second exon that encodes the peptide binding part of the MHC molecule was sequenced for DLA-DRB1 (270 bp), DLA-DQA1 (246 bp) and DLA-DQB1 (267 bp) genes. The genes are located close together on canine chromosome 12 in the above-mentioned order [33]. The distance between DLA-DRB1 and DLA-DQA1 is $56.6 \mathrm{~kb}$, while the distance between DLA-DQA1 and DLA-DQB1 is $77.7 \mathrm{~kb}$. A previously established protocol [13] was used to sequence the loci in a cohort of 253 dogs. In addition, data from 41 families with 192 puppies from an earlier study by Kennedy et al. [34] plus a further 12 families with 64 puppies from Rowan [35] were included. Microsatellite genotyping method for MHC genotyping was used for one mixed-breed group of six families including 37 offspring [35]. In this method, dogs were genotyped using microsatellites CFA12-3 (dinucleotide), CFA12-4 (tetranucleotide), and CFA12-9 (dinucleotide), which are linked with the studied DLA loci [36]. In addition, parental dogs were genotyped by sequencing to confirm the MHC genotypes. 


\section{MHC allele and haplotype assignment}

MHC alleles were determined from the sequence data using MatchTools and MatchTools Navigator (Applied Biosystems) programs. MatchTools includes a library of canine MHC alleles and compares a homozygous or heterozygous sequence pair to the allele sequences in the library. Two puppies from the data by Kennedy et al. [34] had a recombinant allele in one locus and that locus was excluded from the analyses in those individuals.

Usually, the MHC alleles from different loci are inherited as haplotypes due to strong linkage disequilibrium (LD, non-random association of alleles between different loci). Three-locus haplotypes consisting of the DLADRB1, DLA-DQA1 and DLA-DQB1 alleles were defined in three steps: 1) haplotypes were determined for homozygous individuals, 2) haplotypes for heterozygous individuals were inferred based on the haplotypes observed in the homozygous individuals and 3) rare haplotypes, detected only in the heterozygous individuals, were identified by subtraction of haplotypes determined in the previous steps [14]. In 35 families, missing parental haplotypes were inferred based on the haplotypes of the offspring and the other parent. The DLA-DQA1 alleles of three parents and the DLA-DQB1 allele of one parent were inferred based on the family members' alleles.

In the microsatellite-based genotyping of the DLA alleles, the microsatellite data were analysed with Genotyper 3.7 (Applied Biosystems). The microsatellite alleles were matched with the DLA haplotypes in the dogs that had been genotyped by both methods. After identifying the corresponding microsatellite alleles and DLA haplotypes, the DLA haplotypes were assigned to individuals that had been genotyped only with microsatellites.

\section{MHC supertype classification}

MHC supertype classification was developed to find out if prenatal selection acts at that level. MHC alleles in the $\beta$ loci, DLA-DRB1 and DLA-DQB1, were classified into MHC supertypes based on the chemical properties of the amino acid sequences in the putative peptidebinding region (PBR). The classification was done for officially named canine MHC alleles from the MatchTools library comprising alleles from different canine species, mostly from dog and grey wolf (Canis lupus). Altogether 205 DLA-DRB1 alleles and 100 DLA-DQB1 alleles were included.

Since the supertype classification of alleles is based on the shared properties in peptide binding, the putative PBR sites were selected according to human PBR sites proposed by Doytchinova and Flower [37]. Only polymorphic PBR sites were included in the analyses; 19 sites in DLA-DRB1 (codon positions: 9, 11, 13, 26, 28, 30, 38, $47,56,57,60,61,67,70,71,74,78,86$ and 90$)$ and 15 sites in DLA-DQB1 (codon positions: 9, 13, 26, 28, 30,
37, 38, 47, 57, 67, 70, 71, 74, 77 and 85). Also 37 canine DLA-DQA1 alleles were checked for polymorphisms in putative PBR sites but all 10 sites were monomorphic and thus the locus was excluded from the supertype classification.

Chemical properties of the amino acids in PBR sites were described using five $\mathrm{z}$-descriptors: $\mathrm{z}_{1}$ (hydrophobicity), $\mathrm{z}_{2}$ (steric bulk), $\mathrm{z}_{3}$ (polarity), $\mathrm{z}_{4}$ and $\mathrm{z}_{5}$ (electronic effects) [38]. Z-descriptor values were standardised so that their distributions had a mean of zero and a standard deviation of one. All z-descriptor $\times$ polymorphic site combinations were used for estimating Euclidian distances between the different MHC alleles. Based on the Euclidian distances, the alleles were clustered using hierarchical clustering with Ward's method [39] in $\mathrm{R}$ statistical environment v. 3.1.2 [40]. The number of clusters was decided based on the cost of merging clusters in Ward's method. The smallest number of clusters, before a steep increase in the cost of merging clusters (variance within clusters), was chosen. To study which PBR sites had most influence in the clustering and to visualise the relations of the supertype clusters, a principal component analysis (PCA) was conducted using the prcomp function in $\mathrm{R}$.

\section{Segregation analyses}

To study whether the DLA-DRB1, DLA-DQA1 and DLA-DQB1 alleles follow Mendelian inheritance in dog families, heterozygosity analyses were conducted in $\mathrm{R}$ statistical environment v. 3.1.2 [40]. There are four possible mating cross types where differences in expected and observed numbers of heterozygotes can be observed: $A_{1} A_{1} \times A_{1} A_{2}, \quad A_{1} A_{2} \times A_{1} A_{1}, \quad A_{1} A_{2} \times A_{1} A_{2}$ and $A_{1} A_{2} \times$ $A_{1} A_{3}$ (where: mother $\times$ father, and the alleles $A_{1} \neq A_{2} \neq A_{3}$ are composites of different observed alleles). In our dataset, altogether 74 (DLA-DRB1 and DLA-DQB1) and 83 (DLA-DQA1) families including 293 (DLA-DRB1), 313 (DLA-DQA1) and 295 (DLA-DQB1) puppies met this criterion. The numbers of heterozygous and homozygous offspring were counted within each mating cross type. The expected numbers of heterozygous and homozygous offspring were calculated based on the Mendelian inheritance model and compared to observed numbers of offspring using Pearson's chi-squared test with Monte Carlo simulated $p$-value (2 000 repeats). Chi-squared test statistics were also combined over the mating cross types in each locus to examine the overall deviation from the expected number of the heterozygotes.

The analyses were conducted at locus and three-locus haplotype levels for the entire data, and separately for different breeds/groups and sexes to identify the potential breed or sex related differences in the inheritance patterns. Information about sex was available for 381 offspring. In addition, purebred and mixed-breed dogs were analysed separately. Segregation analyses were also 
done for the supertype classes and separately for each polymorphic PBR site in the DLA-DRB1 and DLA-DQB1 loci. To correct for multiple testing, a false discovery rate ( $q$-value [41]) was estimated for $p$-values of the combined chi-squared tests of the different mating cross types over the PBR sites in one locus.

Two haplotypes that were found in four breeds/groups carried a duplicated DLA-DQB1 locus (DLA-DQB1*01303 and DLA-DQB1*01701 [34]). Since it is unclear whether both copies are expressed or not, analyses were conducted including all individuals and separately for the families with (number of puppies $=51 \quad(\mathrm{DRB} 1) / 59 \quad(\mathrm{DQA} 1) / 53$ $(\mathrm{DQB1}))$ and without (number of puppies $=242(\mathrm{DRB} 1$ \& DQB1)/254 (DQA1)) the duplicated locus. When included in the analyses, the duplicated locus was handled as one allele (DLA-DQB1*013017). In the analyses for the DLA-DQB1 locus with supertype classification and for each PBR site, all families with duplicated locus were excluded.

\section{Maternal effect}

We studied the maternal effect on the MHC class II allele transmission in the families where the offspring may inherit the maternal shared or paternal non-shared allele from their father: $A_{1} A_{2} \times A_{1} A_{3}$ and $A_{1} A_{1} \times A_{1} A_{2}$. We tested the distribution between the expected and observed numbers of the shared and non-shared alleles inherited from father with Pearson's chi-squared test with Monte Carlo simulated $p$-value (2 000 repeats).

There is some evidence in man, that the mother's heterozygosity may influence the offspring heterozygosity - in Amerindian families only the heterozygous mothers' had increased proportion of heterozygous offspring [17]. So, to discover whether the mother's heterozygosity has an effect on the heterozygosity of the offspring in dogs, families with heterozygous mothers were joined for analyses. The maternal effect was studied in the total dataset and in the same subsets as described in section 2.4.

\section{Results}

\section{MHC alleles and haplotypes}

Altogether 28 DLA-DRB1, 11 DLA-DQA1 and 23 DLADQB1 alleles organised in 45 three-locus haplotypes were found in the dog sample (Table 2). There were some common haplotypes that were found in about half of the breeds/groups studied but rare unique haplotypes were also detected in many breeds. Three new DLA-DRB1 alleles were found among the 253 dogs sequenced for this study. The allele sequences have been submitted to NCBI GenBank with the accession numbers: JQ904819.1, FM246834, and FM246838.

\section{Supertype classification}

The DLA-DRB1 alleles were divided into five supertype classes and the DLA-DQB1 alleles were divided into nine supertype classes using hierarchical clustering method. The number of classes was based on the cost of merging clusters (Additional file 2: Figures S1-S2). Supertype clustering trees for the canine DLA-DRB1 and DLA-DQB1 alleles are shown in Figs. 1 and 2 and the supertype clustering trees with allele names are shown in Additional file 3: Figures S3-S4. Complete lists of the alleles in each supertype class are in Additional file 4: Table S2.

In humans, each MHC supertype could be described by common fingerprint amino acids that were shared within each supertype class [37]. In canines, such supertype fingerprints could not be detected, since amino acids were shared, to some extent, also between different supertypes. Based on the three first PCs the PBR sites 1, 5, 6, and 9 in DLA-DRB1, and 4, 9, 10, 12, and 15 in DLA-DQB1 had the largest impact on the supertype clustering ( $\mathrm{PC} 1$ vs. $\mathrm{PC} 2$ in Additional file 5: Figures S5-S6). At these sites, there were from two to seven (DLA-DRB1) and from three to five (DLA-DQB1) different amino acids - a similar level of diversity compared to other PBR sites.

\section{MHC genotype proportions in the offspring}

At the locus level, deviation from the Mendelian expectations was not observed in the genotype proportions of the dog families. When examined for all of the families and different mating cross types, $x^{2}$ varied between 0.02 and $2.81(\mathrm{df}=1)$ and $p$-value varied between 1 and 0.13 in all MHC loci (Fig. 3 and Additional file 6: Table S3). Accordingly, the combined chi-squared analyses over mating cross types within each locus did not show any deviation from the expected heterozygosity and the combined $\chi^{2}$ varied between 2.28 and 3.09 $(\mathrm{df}=1)$ and $p$-value varied between 0.69 and 0.54 . All of the MHC loci followed the Mendelian inheritance also in different breeds, in purebreds, in mixed-breed dogs, in both sexes and in families with or without the duplicated DLA-DQB1 locus, when they were analysed separately. No deviation from the expected heterozygosity was detected either at the three-locus haplotype level (Additional file 6: Table S3) or using supertype classification (Fig. 4).

The genotype proportions in the offspring were also studied in each polymorphic PBR site. Figure 5 shows both $p$ - and $q$-values for the combined chi-squared statistics over the different mating cross types in site-wise segregation analyses. After correcting for multiple testing, the PBR sites 10-12 in DLA-DRB1 showed a slight deviation from the expected heterozygosity, with site 12 having the smallest $q$-value $(q=0.15)$. The PBR 
Table 2 Three-locus MHC haplotypes and their frequencies in the studied dog breeds and mixed-breed groups

\begin{tabular}{|c|c|c|c|c|c|c|c|c|c|c|c|c|c|c|c|c|}
\hline DRB1 & DQA1 & DQB1 & $\mathrm{AH}^{1}$ & $\mathrm{AH}^{2}$ & $\mathrm{CB}^{1}$ & $\mathrm{CB}^{2}$ & $\mathrm{ECS}^{2}$ & $\mathrm{GR}^{2}$ & ISD & $\mathrm{KKH}$ & $\mathrm{KL}$ & LW & $\mathrm{NF}^{2}$ & $\mathrm{RRB}^{2}$ & SA & $\mathrm{SBT}^{2}$ \\
\hline 10301 & 00101 & 00802 & & & & & & & & & 0.28 & & & & & \\
\hline 00101 & 00101 & 00201 & 0.15 & & 0.24 & 0.22 & & & 0.08 & & & & & 0.30 & 0.02 & 0.35 \\
\hline 00101 & 00101 & 03601 & & & & & & & & & & 0.12 & & & 0.02 & \\
\hline 00101 & 00101 & 03603 & & & & & & & & & & 0.12 & & & & \\
\hline 00103 & 00101 & 00201 & & & & 0.06 & & & & & & & & & & \\
\hline 00201 & 00901 & 00101 & & 0.25 & & & & & & & & & 0.17 & & & \\
\hline 00601 & 005011 & 00701 & 0.17 & 0.25 & & 0.01 & 1.00 & & & & & & 0.08 & 0.18 & 0.05 & \\
\hline 00601 & 005011 & 02001 & & & & & & 0.12 & & & & & 0.33 & & & \\
\hline 00901 & 00101 & 008011 & 0.32 & & & & & & & 0.42 & 0.03 & 0.47 & & & 0.02 & \\
\hline 010011 & 00201 & 01501 & & & & 0.06 & & & & & & & & & & \\
\hline 01101 & 00201 & 01303 & & & & & & & & 0.04 & & & & & & \\
\hline 01102 & 00201 & 01303 & & & & & & & 0.08 & & & & & & & \\
\hline 01201 & 00401 & $013017^{a}$ & & 0.33 & 0.51 & & & & & & & & 0.19 & & 0.01 & \\
\hline 01201 & 00401 & 01303 & & & & & & 0.42 & & & & & & & & \\
\hline 01301 & 00101 & 00201 & & & & & & & 0.19 & & & & 0.04 & 0.25 & & 0.46 \\
\hline 01301 & 00601 & 02002 & & & & & & & & & & & & & 0.07 & \\
\hline 01501 & 00901 & 00101 & & & & & & & & & & & & & & 0.19 \\
\hline 01501 & 00601 & 02301 & 0.24 & & & 0.31 & & 0.19 & 0.23 & 0.03 & & & 0.04 & & & \\
\hline 01501 & 00601 & 05701 & 0.03 & & & & & & & & & & & & & \\
\hline 01501 & 00601 & 00301 & & & & & & & 0.12 & & & & & & & \\
\hline 01501 & 00601 & 02201 & & & & & & & & & 0.30 & & & & & \\
\hline 01501 & 00601 & 02002 & & & & 0.07 & & & & & & & & & & \\
\hline 01501 & 00401 & $013017^{a}$ & & & & & & & & & & & & & 0.03 & \\
\hline 01501 & 012011 & 03501 & & & & & & & & & & & & & 0.01 & \\
\hline 01501 & 00601 & 01101 & & & & & & & & 0.18 & & 0.09 & & & & \\
\hline 01502 & 00601 & 02301 & & & & & & & & & 0.22 & 0.03 & & & & \\
\hline 01503 & 00601 & 00301 & & & & & & & 0.08 & & & & & & & \\
\hline 01503 & 00601 & 02304 & & & & & & & & 0.07 & & & & & & \\
\hline 01801 & 00101 & 00802 & 0.03 & & 0.24 & & & & & & & & & 0.14 & 0.07 & \\
\hline 02001 & 00401 & 01303 & & & & & & 0.27 & & 0.09 & & & 0.04 & 0.01 & 0.42 & \\
\hline 02301 & 00301 & 00501 & & & & & & & 0.23 & 0.18 & & & & & & \\
\hline 03001 & 00601 & 00301 & & & & & & & & & & & 0.03 & & & \\
\hline 04001 & 01001 & 01901 & & 0.17 & & & & & & & & & & & & \\
\hline 07001 & 01801 & 05001 & 0.06 & & & & & & & & & & & & & \\
\hline 07401 & 005011 & 00701 & & & & & & & & & 0.16 & & & & 0.02 & \\
\hline 07401 & 00101 & 00201 & & & & & & & & & & & & & 0.01 & \\
\hline 07601 & 00601 & 02301 & & & & 0.25 & & & & & & & & & & \\
\hline 07801 & 00401 & 01303 & & & & & & & & & & & & & 0.08 & \\
\hline 08001 & 00402 & 01303 & & & & & & & & & & & & & 0.01 & \\
\hline 09401 & 00101 & 008011 & & & & & & & & & & 0.18 & & & & \\
\hline 09501 & 00301 & 05401 & & & & & & & & & & & & & 0.05 & \\
\hline
\end{tabular}


Table 2 Three-locus MHC haplotypes and their frequencies in the studied dog breeds and mixed-breed groups (Continued)

\begin{tabular}{|c|c|c|c|c|c|c|c|c|c|c|c|c|c|c|c|c|}
\hline 09501 & 00101 & 008011 & & & & & & & & & & & & & 0.02 & \\
\hline 09501 & 00901 & 00101 & & & & & & & & & & & & & 0.02 & \\
\hline 09701 & 00601 & 02002 & & & & & & & & & & & & & 0.04 & \\
\hline \multirow[t]{3}{*}{09801} & 00402 & 02301 & & & & & & & & & & & & & 0.01 & \\
\hline & & NA & & & & 0.02 & & & & & & & 0.08 & 0.12 & 0.01 & \\
\hline & & $n$ & 39 & 6 & 49 & 116 & 8 & 13 & 13 & 37 & 138 & 17 & 66 & 52 & 83 & 13 \\
\hline
\end{tabular}

$A H^{1}$ Alaskan Husky, $A H^{2}$ Alaskan Husky, $C B^{1}$ Mixed-breed, $C B^{2}$ Mixed-breed, $E C S^{2}$ English Cocker Spaniel, $G R^{2}$ Golden Retriever, ISD Icelandic Sheepdog, $K K H$ Kooikerhondje, KL Kromfohrlander, $L W$ Lowchen, $N F^{2}$ Newfoundland, RRB ${ }^{2}$ Rhodesian Ridgeback, SA Saluki, SBT ${ }^{2}$ Staffordshire Bull Terrier $n=$ number of individuals (all litter-parents combinations)

a Duplicated DLA-DQB1 locus, alleles DLA-DQB1*01303 and DLA-DQB1*01701

${ }^{1}$ Rowan [35], ${ }^{2}$ Kennedy et al. [34]

sites 10,11 and 12 correspond to codon positions 57, 60 and 67 in the data. More detailed examination of the PBR site 12 revealed excess of heterozygous offspring from the mating cross type $\mathrm{A}_{1} \mathrm{~A}_{2} \times \mathrm{A}_{1} \mathrm{~A}_{1}\left(\chi^{2}=\right.$ 9.48, $\mathrm{df}=1, p=0.004)$. The site was polymorphic for three amino acids: leucine, isoleucine and phenylalanine. All three amino acids are hydrophobic, but phenylalanine differs from the other two by having an aromatic side chain. Also the PBR site 10 showed excess of heterozygosity $\left(\mathrm{A}_{1} \mathrm{~A}_{2} \times \mathrm{A}_{1} \mathrm{~A}_{1}, \chi^{2}=4.46, \mathrm{df}=1, p=0.04\right)$ but the PBR site 11 showed excess of homozygosity $\left(\mathrm{A}_{1} \mathrm{~A}_{2} \times \mathrm{A}_{1} \mathrm{~A}_{2}, \chi^{2}=4.95, \mathrm{df}=1, p=0.04\right)$. In DLA-DQB1, no PBR sites showed deviation from the expected heterozygosity (Fig. 5).

\section{Maternal effect}

To study the putative prenatal maternal effect, allele transmission was studied in the families in which parents shared one allele and the offspring could inherit the maternal shared or paternal non-shared allele from their father. The puppies tended to inherit the maternal shared allele $\left(A_{1}\right)$ from their father for DLA-DRB1 in mating cross type $A_{1} A_{2} \times A_{1} A_{3}$ more often than expected

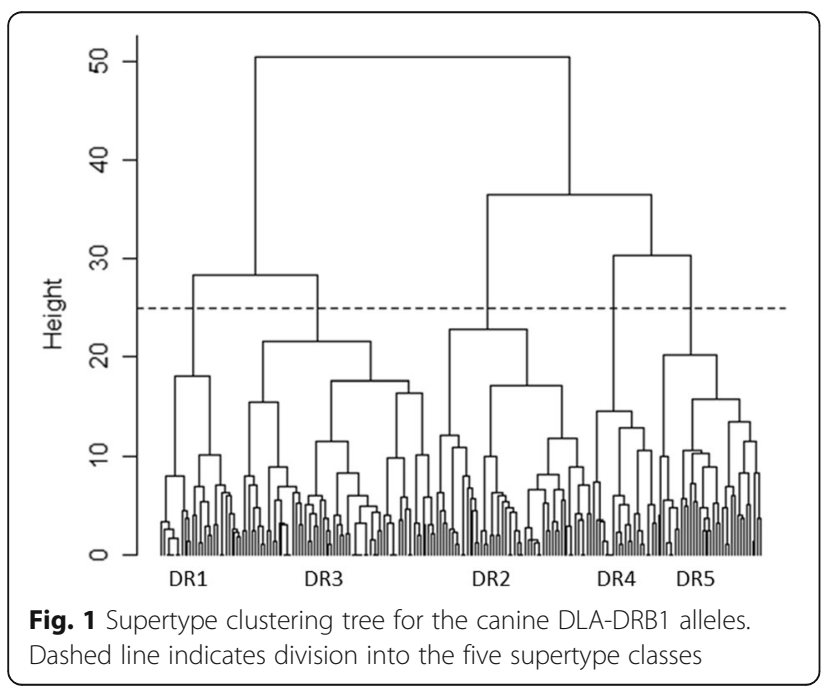

$\left(\chi^{2}=3.02, \mathrm{df}=1, p=0.09\right.$; Fig. 6 and Additional file 6: Table S3). This type of mating included the majority of the families in this analysis. However, no strong deviations from the expected allele proportions were found either when using the allele or supertype classification in the analyses. The maternal shared and nonshared allele were equally often inherited from the father also in different breeds, in both sexes and in families with or without the duplicated DLA-DQB1 locus.

When the heterozygous mothers were combined for segregation analyses, mother's heterozygosity did not affect the offspring heterozygosity neither at the allele nor at the supertype level (Additional file 6: Table S3).

\section{Discussion}

We have studied prenatal selection in dogs by analysing the segregation of the MHC class II alleles in purebred and mixed-breed families. Natural selection targeting the MHC may be limited in the pet dogs due to their protected environment. One potential phase of natural selection is during foetal development which is less strongly affected by human action or breeding choices. However, we did not detect deviations from the Mendelian

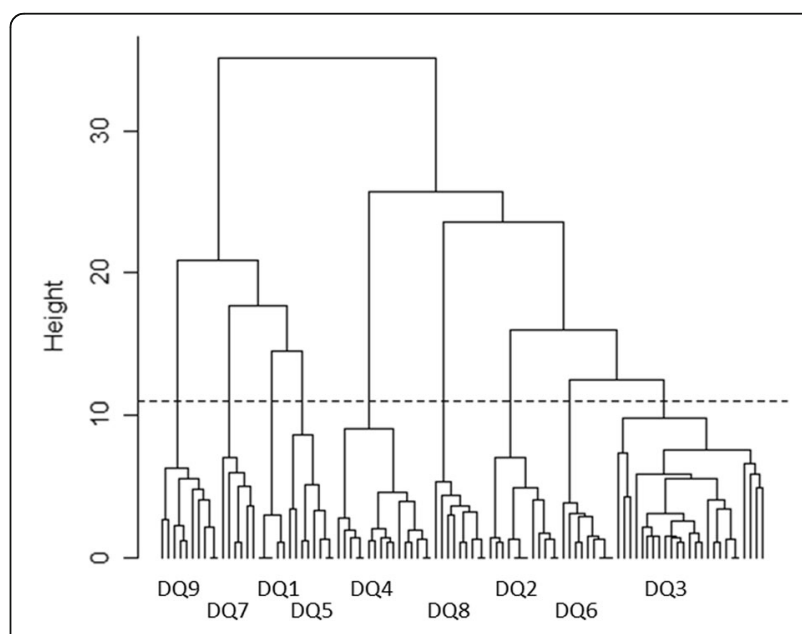

Fig. 2 Supertype clustering tree for the canine DLA-DQB1 alleles. Dashed line indicates division into the nine supertype classes 

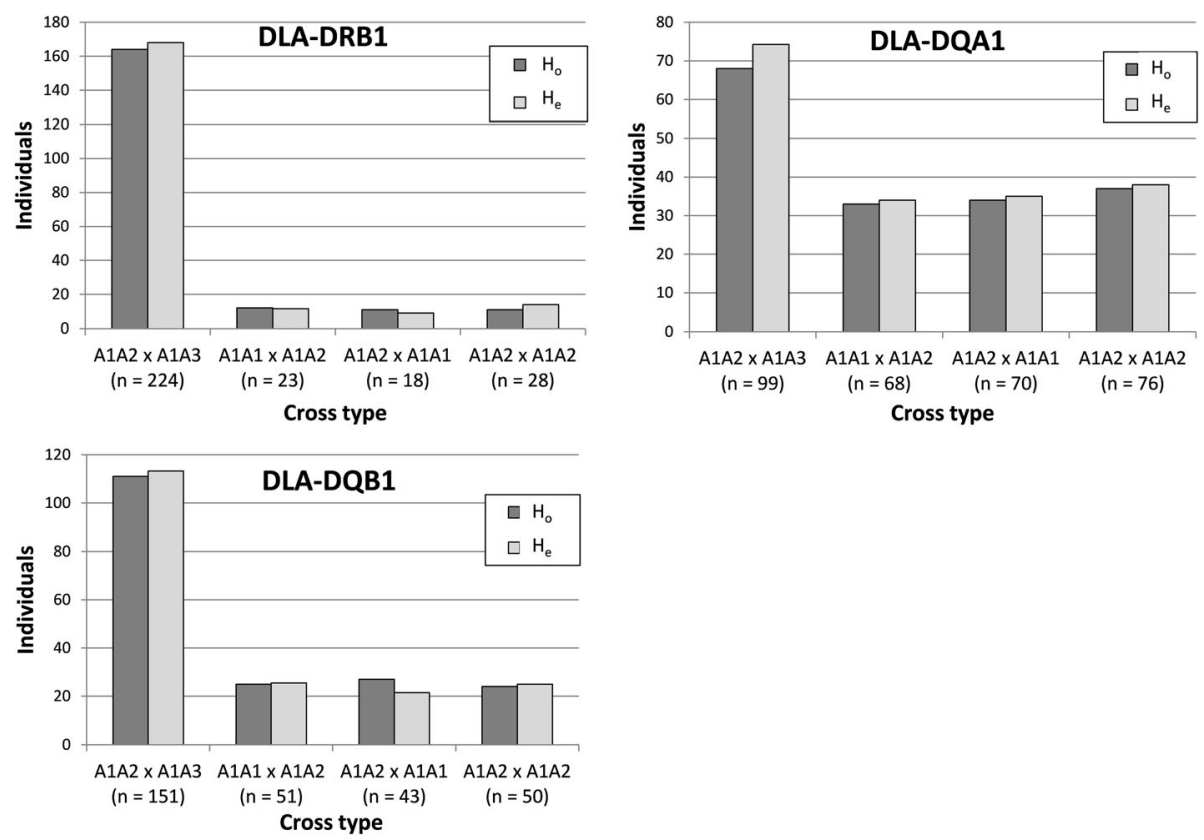

Fig. 3 The observed $\left(H_{0}\right)$ and expected $\left(H_{e}\right)$ numbers of the heterozygous offspring from different mating cross types for the DLA-DRB1, DLA-DQA1 and DLA-DQB1 loci ( $n=$ total number of offspring within the cross type)

segregation pattern at allele, three-locus haplotype, or supertype levels.

At least around 200 DLA-DRB1, 100 DLA-DQB1 and 40 DLA-DQA1 alleles have been found in canines. Obviously there is some functional overlap in such a large number of alleles. In humans, it has been shown that the MHC class II alleles have more shared peptide binding repertoire than the MHC class I alleles [42]. Clustering the MHC alleles into supertypes is one way to reach a meaningful level of functionality and variation for studying the natural selection in the MHC. We have generated the first canine supertype classification for the DLA-DRB1 and DLA-DQB1 alleles. The DLADQA1 alleles were not included, since they were not polymorphic on the putative PBR sites proposed by
Doytchinova and Flower [37]. This might have only minor effect to our results, since in humans it has been shown that the HLA-DQA1 alleles did not contribute to the supertype clustering when they were analysed together with the HLA-DQB1 alleles to compose a single functional unit [37]. In this study, the number of the supertype clusters (five and nine) was relatively low, and based on in silico analysis of amino-acids. Functional studies, where the peptide binding repertoires of the different MHC alleles could be tested, would greatly benefit the extraction of a biologically meaningful number of supertype clusters [31, 42].

The supertype-clustered MHC alleles can be used in selection analyses instead of the sequence-based alleles. However, we did not find patterns suggesting prenatal
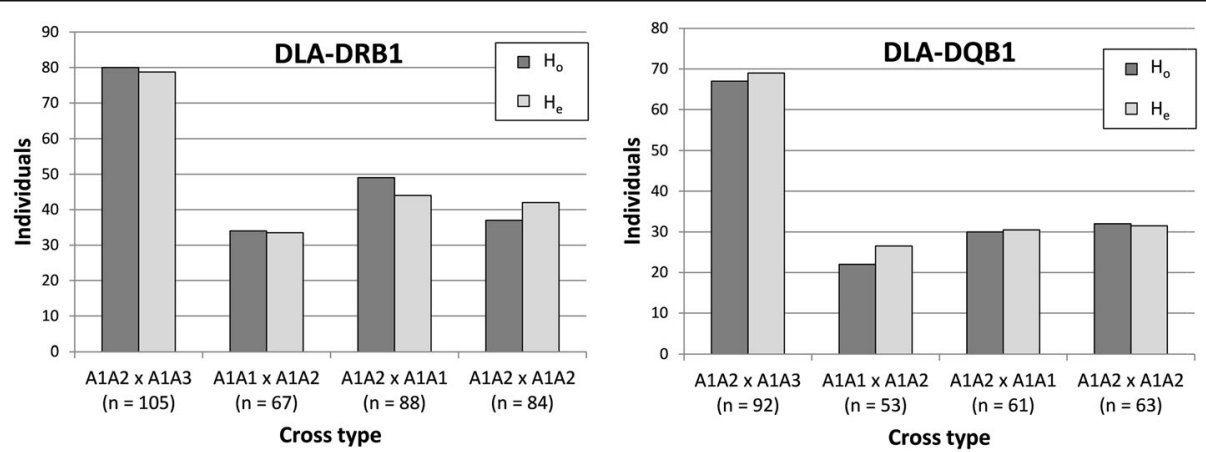

Fig. 4 The observed $\left(H_{0}\right)$ and expected $\left(H_{e}\right)$ numbers of the heterozygous offspring from different mating cross types for the DLA-DRB1 and DLA-DQB1 supertypes ( $n=$ total number of offspring within the cross type) 

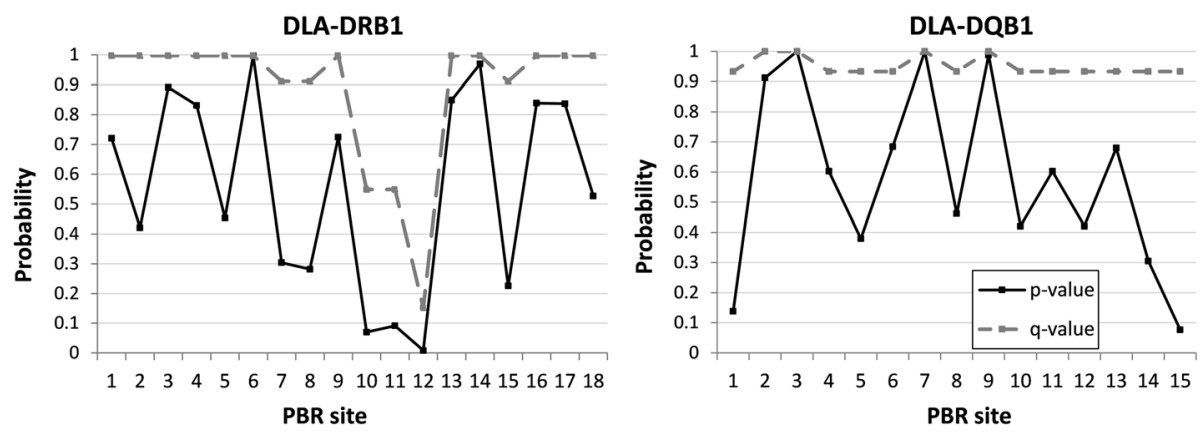

Fig. 5 The $p$ - and $q$-values for the combined chi-squared statistics testing the ratio of the observed and expected heterozygous offspring for each PBR site in the DLA-DRB1 and DLA-DQB1 loci

selection at supertype level in the studied dog families. Previously, natural selection has been detected at the supertype level e.g. in the higher male heterozygosity of newborn humans [43] and in the non-random association of the alleles within DRB-haplotypes of baboons [31]. Parasite and disease resistance has also been associated with MHC supertype level classes in various species [30, 32]. These findings suggest that supertype classification reaches a level of functionality that may be missed using individual alleles in the selection analyses.

In humans, some small isolated populations have shown non-Mendelian proportions of MHC genotypes in offspring [17] or MHC associated foetal loss [8]. However, often in studies of larger human populations no deviation from the expected proportions has been found in the MHC loci $[1,44]$. The lack of selection in the modern large human populations might be a consequence of improved health care [45] which also applies to dogs but is likely to mostly affect selective factors after birth and to less affect maternal-foetal interactions. In this study, the puppies were sampled in varying ages and the age of the dog was not known in all cases.
Thus, it is possible that a small degree of postnatal selection has occurred.

We found that the dog puppies were more heterozygous than expected in respect to the PBR-site 12 of DLA-DRB1 for the mating cross type $A_{1} A_{2} \times A_{1} A_{1}$. When half of the offspring are expected to be heterozygous and half homozygous, the selection coefficient $(s)$ can be estimated with:

$$
s=1-N_{i i} /\left(N_{i j}+1\right)
$$

where $N_{i i}$ is the observed number of the homozygous offspring and $N_{i j}$ is the observed number of the heterozygous offspring [46]. The observed excess of the heterozygous individuals at the PBR-site 12 of DLA-DRB1 would require strong selection against homozygotes: $s=1-38 /(70+1)=0.465$. Selection of similar strength, $s=0.462$ over two MHC loci, has been estimated to favor heterozygotes in human populations [17]. However, because the MHC expression in canine fertilisation and pregnancy is not well known, further studies are required to investigate the exact cause of the observed deviation,

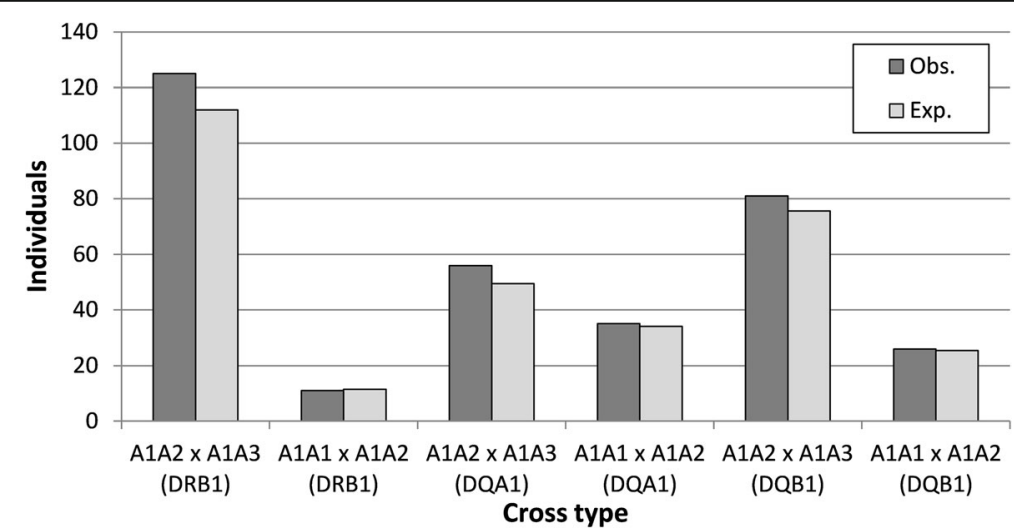

Fig. 6 The observed (Obs.) and expected (Exp.) numbers of offspring that inherited the maternal shared allele from their father for DLA-DRB1, DLA-DQA1 and DLA-DQB1 loci 
before reaching further conclusions about the selective advantage of heterozygosity on the PBR-site 12. The amino acids at the PBR-site 12 were shared by different alleles and supertypes, which explains why we did not detect the signal of this deviation at the higher levels of analyses.

Our data consisted of ten dog breeds, that have been bred for e.g. hunting, herding, and companion purposes, and mixed-breed groups. The sampling of numerous breeds had the advantage of representing the species more widely, but as a downside, it also caused individual alleles to have low frequencies in the highly polymorphic MHC loci. To gain statistical power, we classified the mating cross types by the alleles the parents shared without keeping the information of allele identity. Simultaneously, we compromised the putative effects of individual alleles. We estimated the statistical power of the $x^{2}$ test to distinguish between the expected and observed numbers of puppies at the $p=0.05$ level. Using the sample sizes shown in Fig. 3 for each mating cross type and locus, $s$ should approximately be above $0.3-0.6$ to be detected. Values this high indicate very strong selection and are rarely observed in natural populations. It is possible that there are breed-specific differences, which we were not able to recognise due to the small breed-wise sample size, particularly when the sample was further divided into mating cross types. As shown in earlier studies [17], small sample size can hinder us from drawing final conclusions about the differences in the post-copulatory selection in dog breeds, especially if selection is not very strong.

Due to the high LD within the MHC region, MHC class II alleles could show the effect of prenatal selection even if the class II alleles are not expressed in the placenta. Other prenatally selected loci that are in LD with the classical MHC loci may contribute to the maintenance of the high polymorphism in the extended MHC region [47]. Simultaneously, this may lead to apparent segregation distortion also in the classical MHC genes. For example, Laurent et al. [48] detected a signature of disassortative mating using single nucleotide polymorphisms (SNPs) in the extended human MHC region (3.6 Mb), and concluded that it was caused by multiple MHC related genes rather than the classical MHC genes. Mate choice is seldom possible in dogs, but post-copulatory selection may, in a similar way, be caused by several MHC-linked genes.

The OR genes may influence both mate selection and the success of spermatozoa in the fertilisation [47]. Previously observed non-Mendelian segregation of MHC alleles in primates may be caused by selection targeted to MHC-linked OR genes. If that was the case, the missing selection in the MHC class II loci of our dog families is easily explained by the fact that no OR genes have this far been localised in the major $3 \mathrm{Mb} \mathrm{MHC}$ region on the dog chromosome 12 or elsewhere on chromosome 12 [33, 49]. Dog spermatozoa do express OR genes [50], but putatively MHC-linked OR genes are only found in the minor $600 \mathrm{~kb}$ MHC class I region located on dog chromosome 35 [33].

\section{Conclusions}

In summary, we found some evidence of post-copulatory selection in pet dogs at nucleotide site level, but not at locus, three-locus, or supertype levels in MHC loci. It is possible that there is weaker selection affecting other sites and levels, but our sample, which only spanned one generation, was not large enough to pick up the signal [1]. Since selection has modified the MHC diversity over a long time period, even weak selection may have been sufficient to preserve the diversity.

To our knowledge, this was the first study of prenatal selection in $\operatorname{dog}$ MHC loci and we have some suggestions how to proceed with future studies. In further examinations of $\mathrm{MHC}$ related prenatal selection in canines, a comprehensive study including mate choice, segregation of alleles and litter size would be interesting. Future studies would also benefit from using dogs from more primitive circumstances and, for comparison, their wild wolf relatives.

\section{Additional files}

Additional file 1: Table S1. MHC genotype data and sample information for the dog families. (XLSX 53 kb)

Additional file 2: Figures S1 and S2. Merging cost showing the amount of variance within clusters in Ward's method. This information was used in deciding the number of supertype clusters in DLA-DRB1 (Figure S1) and DLA-DQB1 (Figure S2) loci. (ZIP 3 kb)

Additional file 3: Figures S3 and S4. Supertype clustering trees for the canine DLA-DRB1 (Figure S3) and DLA-DQB1 (Figure S4) loci with allele names included. The supertype clusters are coloured differently. (ZIP 12 kb)

Additional file 4: Table S2. Supertype classification allele data and all alleles in the dog family data. (XLSX $25 \mathrm{~kb}$ )

Additional file 5: Figures S5 and S6. Biplots of the first two PCs in supertype clusters of DLA-DRB1 (Figure S5) and DLA-DQB1 loci

(Figure S6). The biplots were done using the ggbiplot function in R. Ellipses show Normal contour lines with $68 \%$ probability for each cluster. (ZIP $20 \mathrm{~kb})$

Additional file 6: Table S3. Result table for segregation of heterozygous and homozygous offspring in different mating cross types for the MHC loci, the three-locus haplotype and the MHC supertype levels, heterozygous mothers combined and the numbers of offspring that inherited maternal shared or paternal non-shared allele from their father. (XLSX $12 \mathrm{~kb})$

\section{Abbreviations}

df: degree of freedom; DLA: Dog leucocyte antigen; DNA: Deoxyribonucleic acid; EVT: Extravillous trophoblast cells; $F_{I s}$ : Inbreeding coefficient; $H_{e}$ : Expected number of heterozygotes; HLA: Human leucocyte antigen; $H_{0}$ : Observed number of heterozygotes; LD: Linkage disequilibrium; 
MHC: Major histocompatibility complex; $N_{F}$ : Number of families; NK: Natural killer cells; $N_{O}$ : Number of offspring; OR: Olfactory receptor; PBR: Peptide-binding region; PC(A): Principal component (analysis); s: selection coefficient

\section{Acknowledgements}

We are grateful to Ingerid Hagen and two anonymous reviewers for providing valuable comments on the earlier version of this manuscript. We thank Ranja Eklund for collecting saluki data, breed clubs for collaboration in collecting dog samples in Finland, and George Happ from the University of Alaska at Fairbanks, Alaska, for husky samples. We thank Frances Jury for typing and partial analyses of the MHC microsatellite results for the husky families in Manchester.

\section{Availability of data and material}

The datasets supporting the conclusions of this article are included within the article and its additional files. The new DLA-DRB1 allele sequences have been submitted to NCBI GenBank with accession numbers: JQ904819.1, FM246834, and FM246838.

\section{Authors' contributions}

AKN conducted part of the laboratory work. AKN, LJK and HL collected the data. AKN, LJK and TP implemented the data analyses. AKN, TP and JA wrote the manuscript with editorial input from all authors. All authors approved the final manuscript.

\section{Competing interests}

Lorna Kennedy is Managing Editor on this journal, and was excluded from the Editorial process. Other authors declare no competing interests.

\section{Funding}

This study was partially supported by the Finnish Population Genetics Graduate Programme (AKN), Kone Foundation (AKN), the Academy of Finland (HL: 127065), the Sigrid Juselius Foundation $(\mathrm{HL})$ and the Jane and Aatos Erkko Foundation ( $\mathrm{HL})$.

\section{Consent for publication}

Not applicable.

\section{Ethics approva}

No dog DNA samples were collected specifically for this study.

\section{Author details}

'Department of Genetics and Physiology, University of Oulu, PO Box 3000, Oulu FIN-90014, Finland. ${ }^{2}$ Present address: Centre for Biodiversity Dynamics, Department of Biology, Norwegian University of Science and Technology, NO -7491, Trondheim, Norway. ${ }^{3}$ Centre for Integrated Genomic Medical Research, University of Manchester, Stopford Building, Oxford Road, Manchester M13 9PT, UK. ํDepartment of Veterinary Biosciences, Research Programs Unit, Molecular Neurology, University of Helsinki and Folkhälsan Institute of Genetics, Biomedicum Helsinki, PO Box 63, FIN-00014 Helsinki, Finland.

\section{Received: 28 January 2016 Accepted: 2 October 2016}

Published online: 18 November 2016

\section{References}

1. Garrigan D, Hedrick PW. Perspective: Detecting Adaptive Molecular Polymorphism: Lessons From the MHC. Evolution (N Y). 2003;57:1707-22.

2. Benacerraf $B$. Role of $M H C$ gene products in immune regulation. Science (80-). 1981:212:1229-38.

3. Ehlers A, Beck S, Forbes SA, Trowsdale J, Volz A, Younger R, Ziegler A. MHCLinked Olfactory Receptor Loci Exhibit Polymorphism and Contribute to Extended HLA/OR-Haplotypes. Genome Res. 2000;10:1968-78.

4. Xie T, Rowen L, Aguado B, Ahearn ME, Madan A, Qin S, Campbell RD, Hood L. Analysis of the Gene-Dense Major Histocompatibility Complex Class III Region and Its Comparison to Mouse. Genome Res. 2003;13:2621-36.

5. Clarke B, Kirby DRS. Maintenance of Histocompatibility Polymorphisms. Nature. 1966;211:999-1000.

6. Doherty PC, Zinkernagel RM. Enhanced immunological surveillance in mice heterozygous at the H-2 gene complex. Nature. 1975;256:50-2.
7. Apanius V, Penn D, Slev PR, Ruff LR, Potts W. The nature of selection on the major histocompatibility complex. Crit Rev Immunol. 1997;17:179-224.

8. Ober C. Studies of HLA, fertility and mate choice in a human isolate. Hum Reprod Update. 1999;5:103-7.

9. Penn DJ. The scent of genetic compatibility: sexual selection and the Major Histocompatibility Complex. Ethology. 2002;108:1-21.

10. Fernando MMA, Stevens CR, Walsh EC, De Jager PL, Goyette P, Plenge RM, Vyse TJ, Rioux JD. Defining the Role of the MHC in Autoimmunity: A Review and Pooled Analysis. PLoS Genet. 2008;4:e1000024.

11. Jokinen $P$. Identifying genetic risk factors in canine autoimmune disorders Helsinki: University of Helsinki; 2011.

12. Kennedy $L$, Barnes $A$, Short A, Brown JJ, Lester S, Seddon J, Fleeman L, Francino O, Brkljacic M, Knyazev S, Happ GM, Ollier WER. Canine DLA diversity: 1. New alleles and haplotypes. Tissue Antigens. 2007;69:272-88.

13. Niskanen AK, Kennedy LJ, Ruokonen M, Kojola I, Lohi H, Isomursu M, Jansson E, Pyhäjärvi T, Aspi J. Balancing selection and heterozygote advantage in major histocompatibility complex loci of the bottlenecked Finnish wolf population. Mol Ecol. 2014;23:875-89.

14. Kennedy $L$, Angles JM, Barnes A, Carmichael LE, Radford AD, Ollier WER Happ GM. DLA-DRB1, DQA1, and DQB1 alleles and haplotypes in North American gray wolves. J Hered. 2007;98:491-9.

15. Niskanen AK, Hagström E, Lohi H, Ruokonen M, Esparza-Salas R, Aspi J, Savolainen P. MHC variability supports dog domestication from a large number of wolves: high diversity in Asia. Heredity (Edinb). 2013;110:80-85.

16. Wedekind C, Chapuisat M, Macas E, Rülicke T. Non-random fertilization in mice correlates with the MHC and something else. Heredity (Edinb). 1996;77:400-9.

17. Black F, Hedrick P. Strong balancing selection at HLA loci: evidence from segregation in South Amerindian families. Proc Natl Acad Sci U S A. 1997;94(November):12452-6.

18. Knapp LA, Ha JC, Sackett GP. Parental MHC antigen sharing and pregnancy wastage in captive pigtailed macaques. J Reprod Immunol. 1996:32:73-88.

19. Billington WD. Influence of Immunological Dissimilarity of Mother and Foetus on Size of Placenta in Mice. Nature. 1964;202:317-8.

20. Warning JC, McCracken SA, Morris JM. A balancing act: mechanisms by which the fetus avoids rejection by the maternal immune system. Reproduction. 2011:141:715-24.

21. Kovats S, Main EK, Librach C, Stubblebine M, Fisher SJ, DeMars R. A class I antigen, HLA-G, expressed in human trophoblast. Science (80-). 1990;248:220-3.

22. King A, Boocock C, Sharkey AM, Gardner L, Beretta A, Siccardi AG, Loke YW. Evidence for the expression of HLA-C class I mRNA and protein by human first trimester trophoblast. J Immunol. 1996;156:2068-76.

23. Trowsdale J, Betz AG. Mother's little helpers: mechanisms of maternal-fetal tolerance. Nat Immunol. 2006;7:241-6.

24. Parham P, Moffett A. Variable NK cell receptors and their MHC class I ligands in immunity, reproduction and human evolution. Nat Rev Immunol. 2013;13:133-44

25. Chazara O, Xiong S, Moffett A. Maternal KIR and fetal HLA-C: a fine balance. J Leukoc Biol. 2011;90:703-16.

26. Moffett A, Loke C. Immunology of placentation in eutherian mammals. Nat Rev Immunol. 2006:6:584-94.

27. Schäfer-Somi S, Beceriklisoy HB, Budik S, Kanca H, Aksoy OA, Polat B, Cetin Y Ay SS, Aslan S. Expression of genes in the canine pre-implantation uterus and embryo: Implications for an active role of the embryo before and during invasion. Reprod Domest Anim. 2008;43:656-63.

28. Schäfer-Somi S, Beceriklisoy H, Walter I, Sabitzer S, Klein D, Kanca H, Kaya D, Agaoglu A, Izgür H, Aslan S. Expression of MHC-I and -II in Uterine Tissue from Early Pregnant Bitches. Reprod Domest Anim. 2009;44:103-8.

29. Sette A, Newman M, Livingston B, McKinney D, Sidney J, Ishioka G, Tangri S, Alexander J, Fikes J, Chesnut R. Optimizing vaccine design for cellular processing, MHC binding and TCR recognition. Tissue Antigens. 2002:59:443-51.

30. Schwensow N, Fietz J, Dausmann KH, Sommer S. Neutral versus adaptive genetic variation in parasite resistance: importance of major histocompatibility complex supertypes in a free-ranging primate. Heredity (Edinb). 2007:99:265-77.

31. Huchard E, Weill M, Cowlishaw G, Raymond M, Knapp LA. Polymorphism, haplotype composition, and selection in the Mhc-DRB of wild baboons. Immunogenetics. 2008;60:585-98. 
32. Sepil I, Lachish S, Hinks AE, Sheldon BC. Mhc supertypes confer both qualitative and quantitative resistance to avian malaria infections in a wild bird population. Proc R Soc B. 2013;280:20130134.

33. Yuhki N, Beck T, Stephens R, Neelam B, O'Brien SJ. Comparative genomic structure of human, dog, and cat MHC: HLA, DLA, and FLA.J Hered. 2007;98:390-9.

34. Kennedy LJ, Barnes A, Short A, Brown JJ, Lester S, Seddon J, Happ GM Ollier WER. Canine DLA diversity: 2. Family studies. Tissue Antigens. 2007;69 Suppl 1:289-91.

35. Rowan D. A time and cost effective method for Dog Leukocyte Antigen (DLA) typing using canine microsatellite markers. Manchester: University of Manchester; 2007.

36. Debenham SL, Hart EA, Ashurst JL, Howe KL, Quail MA, Ollier WER, Binns MM. Genomic sequence of the class II region of the canine MHC: comparison with the MHC of other mammalian species. Genomics. 2005;85:48-59.

37. Doytchinova IA, Flower DR. In silico identification of supertypes for class II MHCs. J Immunol. 2005;174:7085-95.

38. Sandberg M, Eriksson L, Jonsson J, Sjöström M, Wold S. New chemical descriptors relevant for the design of biologically active peptides. A multivariate characterization of 87 amino acids. J Med Chem. 1998:41:2481-91.

39. Ward JH. Hierarchical grouping to optimize an objective function. J Am Stat Assoc. 1963;58:236-44.

40. R core team. R: A language and environment for statistical computing. 2014.

41. Storey JD, Tibshirani R. Statistical significance for genomewide studies. Proc Natl Acad Sci U S A. 2003;100:9440-5.

42. Greenbaum J, Sidney J, Chung J, Brander C, Peters B, Sette A. Functional classification of class II human leukocyte antigen (HLA) molecules reveals seven different supertypes and a surprising degree of repertoire sharing across supertypes. Immunogenetics. 2011;63:325-35.

43. Dorak MT, Lawson T, Machulla HK, Mills Kl, Burnett AK. Increased heterozygosity for MHC class II lineages in newborn males. Genes Immun. 2002;3:263-9.

44. Klitz W, Lo SK, Neugebauer M, Baur MP, Albert ED, Thomson G. A comprehensive search for segregation distortion in HLA. Hum Immunol. 1987;18:163-80.

45. Markow T, Hedrick PW, Zuerlein K, Danilovs J, Martin J, Vyvial T, Armstrong C. HLA polymorphism in the Havasupai: evidence for balancing selection. Am J Hum Genet. 1993;53:943-52.

46. Haldane JBS. The estimation of viabilities. J Genet. 1956;54:294-6.

47. Ziegler A, Santos PSC, Kellermann T, Uchanska-Ziegler B. Self/nonself perception, reproduction and the extended MHC. Self Nonself. 2010;1:176-91

48. Laurent R, Toupance B, Chaix R. Non-random mate choice in humans: insights from a genome scan. Mol Ecol. 2012;21:587-96.

49. Quignon P, Giraud M, Rimbault M, Lavigne P, Tacher S, Morin E, Retout E, Valin A-S, Lindblad-Toh K, Nicolas J, Galibert F. The dog and rat olfactory receptor repertoires. Genome Biol. 2005;6:R83.

50. Vanderhaeghen P, Schurmans S, Vassart G, Parmentier M. Specific repertoire of olfactory receptor genes in the male germ cells of several mammalian species. Genomics. 1997;39:239-46.

\section{Submit your next manuscript to BioMed Central and we will help you at every step:}

- We accept pre-submission inquiries

- Our selector tool helps you to find the most relevant journal

- We provide round the clock customer support

- Convenient online submission

- Thorough peer review

- Inclusion in PubMed and all major indexing services

- Maximum visibility for your research

Submit your manuscript at www.biomedcentral.com/submit

) Biomed Central 